\title{
Pilot Randomized Trial of a Transdisciplinary Geriatric and Palliative Care Intervention for Older Adults With Cancer
}

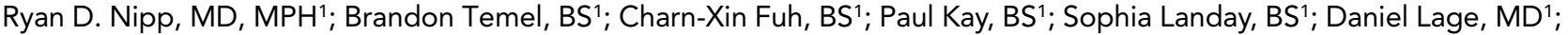 \\ Esteban Franco-Garcia, MD²; Erin Scott, MD²; Erin Stevens, DO²; Terrence O’Malley, MD²,3; Supriya Mohile, MD, MS4; \\ William Dale, MD, PhD'; Lara Traeger, $\mathrm{PhD}^{6}$; Ardeshir Z. Hashmi, MD; Vicki Jackson, MD²; Joseph A. Greer, PhD; \\ Areej El-Jawahri, MD'; and Jennifer S. Temel, MD
}

\begin{abstract}
Background: Oncologists often struggle with managing the unique care needs of older adults with cancer. This study sought to determine the feasibility of delivering a transdisciplinary intervention targeting the geriatric-specific (physical function and comorbidity) and palliative care (symptoms and prognostic understanding) needs of older adults with advanced cancer. Methods: Patients aged $\geq 65$ years with incurable gastrointestinal or lung cancer were randomly assigned to a transdisciplinary intervention or usual care. Those in the intervention arm received 2 visits with a geriatrician, who addressed patients' palliative care needs and conducted a geriatric assessment. We predefined the intervention as feasible if $>70 \%$ of eligible patients enrolled in the study and $>75 \%$ of eligible patients completed study visits and surveys. At baseline and week 12, we assessed patients' quality of life ( $\mathrm{QoL})$, symptoms, and communication confidence. We calculated mean change scores in outcomes and estimated intervention effect sizes (ES; Cohen's $d$ ) for changes from baseline to week 12, with 0.2 indicating a small effect, 0.5 a medium effect, and 0.8 a large effect. Results: From February 2017 through June 2018, we randomized 62 patients $(55.9 \%$ enrollment rate [most common reason for refusal was feeling too ill]; median age, 72.3 years; cancer types: $56.5 \%$ gastrointestinal, $43.5 \%$ lung). Among intervention patients, $82.1 \%$ attended the first visit and $79.6 \%$ attended both. Overall, $89.7 \%$ completed all study surveys. Compared with usual care, intervention patients had less $\mathrm{QoL}$ decrement $(-0.77$ vs -3.84 ; ES $=0.21)$, reduced number of moderate/severe symptoms $(-0.69$ vs +1.04 ; ES = $0.58)$, and improved communication confidence (+1.06 vs -0.80 ; $E S=$ 0.38 ). Conclusions: In this pilot trial, enrollment exceeded $55 \%$, and $>75 \%$ of enrollees completed all study visits and surveys. The transdisciplinary intervention targeting older patients' unique care needs showed encouraging ES estimates for enhancing patients' QoL, symptom burden, and communication confidence.
\end{abstract}

J Natl Compr Canc Netw 2020;18(5):591-598 doi: 10.6004/jnccn.2019.7386

\footnotetext{
${ }^{1}$ Department of Medicine, Division of Hematology and Oncology, and ${ }^{2}$ Department of Medicine, Division of Palliative Care and Geriatric Medicine, Massachusetts General Hospital and Harvard Medical School, Boston, Massachusetts; ${ }^{3}$ Partners Continuing Care, Partners HealthCare System, Boston, Massachusetts; ${ }^{4}$ Department of Medicine, Division of Hematology and Oncology, University of Rochester Medical Center, Rochester, New York; ${ }^{5}$ Department of Supportive Care Medicine, City of Hope National Medical Center, Duarte, California; ${ }^{6}$ Department of Psychiatry, Massachusetts General Hospital and Harvard Medical School, Boston, Massachusetts; and ${ }^{7}$ Department of Internal Medicine and Geriatrics, Cleveland Clinic, Cleveland, Ohio.
}

\section{Background}

Older adults represent a growing population with complex medical problems, including cancer, which disproportionately impacts these individuals. ${ }^{1}$ More than half of newly diagnosed cancers occur in patients aged $>65$ years, and this older population accounts for nearly three-fourths of cancer deaths. ${ }^{1}$ Older adults diagnosed with cancer also experience worse survival outcomes than their younger counterparts, which may result from differential treatment of the geriatric cancer population. ${ }^{2}$ Compared with younger patients, older patients with cancer are more likely to be undertreated and experience earlier discontinuation of treatment for their stage of cancer. ${ }^{3,4}$ Although studies have suggested that poor symptom management and inadequate social support may explain this suboptimal treatment of older patients, more research is needed to address the multifaceted geriatric and palliative care needs of this population. $^{3,4}$

A challenging constellation of medical and psychosocial issues can add to the complexity of caring for older adults with cancer. Older patients experience unique concerns related to their physical function, comorbid conditions, and medication management (ie, geriatric-specific issues), as well as their symptom burden, prognostic understanding, and coping (ie, palliative care issues). ${ }^{5-9}$ Despite the unique health problems of older adults with cancer, interventions targeting the geriatric-specific and palliative care concerns of this population are lacking. ${ }^{7,10-14}$ Importantly, prior work has demonstrated that palliative care interventions improve quality of life (QoL), mood, and possibly even survival for patients with advanced cancer. ${ }^{15-17}$ However, for older adults with cancer, palliative care consultation alone may not fully address all of their concurrent medical and psychosocial comorbidities. ${ }^{18,19}$ The physiologic, psychological, and social support needs of older and younger patients differ, and evidence suggests that older patients with cancer should receive palliative care interventions tailored to their unique needs. ${ }^{5,7,8,18,19}$ In addition, geriatricians have 
developed tools to help assess and manage older patients' distinct, geriatric-specific concerns, but we lack data on whether these interventions effectively address patients' palliative care needs. ${ }^{20}$ Thus, we must develop and test interventions targeting both the geriatric and palliative care issues unique to older adults with cancer.

We conducted a pilot randomized controlled trial (RCT) of a transdisciplinary intervention designed to address the geriatric-specific and palliative care needs of older adults with cancer. We developed the intervention using a transdisciplinary approach, which included integrative and collaborative training to blend the disciplines of geriatrics and palliative care. We sought to determine the feasibility and acceptability of this intervention and to estimate effect sizes for improving patient-reported outcomes. We hypothesized that the transdisciplinary intervention would be feasible to deliver and that patients would find the intervention acceptable. Importantly, data from this study will inform future work by allowing us to estimate effect sizes of the transdisciplinary intervention for improving patients' QoL, symptoms, functional outcomes, communication confidence, and illness perceptions.

\section{Methods}

\section{Study Design and Procedures}

From February 15, 2017, through June 28, 2018, we enrolled patients at Massachusetts General Hospital (MGH) in an unblinded RCT of a transdisciplinary intervention versus usual care (ClinicalTrials.gov identifier: NCT02868112). Trained study staff identified and recruited patients throughout the study period by monitoring the oncology clinic schedules. Following informed written consent, patients completed baseline study measures. After completion, the Office of Data Quality randomly assigned patients 1:1 to receive the transdisciplinary intervention or usual care, stratified by cancer type (gastrointestinal or lung). The Dana-Farber/Harvard Cancer Center Institutional Review Board approved the study protocol.

\section{Participants}

Patients eligible for study participation included those aged $\geq 65$ years and within 8 weeks of a diagnosis of incurable gastrointestinal or lung cancer. We determined whether patients had incurable disease based on chemotherapy order treatment intent designation (palliative vs curative), or documentation from oncology clinic notes in the electronic health record (EHR) if patients were not receiving chemotherapy. Study participants also had to be able to read and respond to study questionnaires in English. Patients who were not planning to receive their longitudinal cancer care at MGH were excluded.

\section{Transdisciplinary Geriatric and Palliative Care Intervention}

Based on a conceptual model of geriatric assessmentdriven interventions in oncology and guideline recommendations, we developed a framework for the transdisciplinary intervention (Figure 1). ${ }^{12-14,21}$ We chose a transdisciplinary approach to intervention development, which included an integrative and collaborative training model that merged the disciplines of geriatrics and palliative care. ${ }^{22}$ Patients assigned to the transdisciplinary intervention participated in 2 in-person visits with a board-certified geriatrician. Visits took place in the geriatricians' clinic, which is not co-located in the cancer center. The first visit occurred within 6 weeks of enrollment and the second visit within 4 to 6 weeks after the initial visit. Before the study began, the geriatricians received 4 one-hour in-person didactic sessions, delivered by geriatricians, palliative care clinicians, and a medical oncologist, focused on the unique geriatric-specific and palliative care issues of an older oncology population (eg, chemotherapy logistics and adverse effects, cancer-specific symptoms and prognosis, collaborating with the oncology team, symptom management, and prognostic disclosure). In addition,

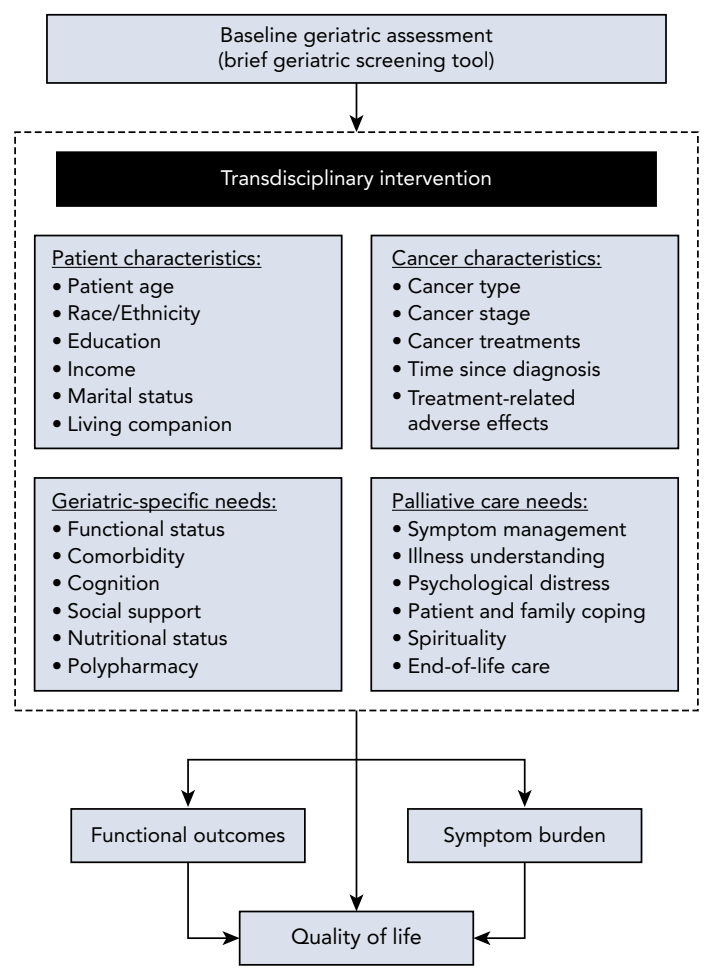

Figure 1. Intervention framework. 
patients completed a brief geriatric screening tool before the first visit, which we then provided to the geriatricians to allow them to tailor the patients' care. The screening tool contained information about patients' self-reported symptom burden, ${ }^{23,24}$ nutrition status, ${ }^{25}$ and vulnerability. ${ }^{26}$ For each visit, we provided the geriatricians with templated notes that the clinicians entered in the EHR, which included topics focused on patients' palliative care needs and issues obtained from geriatric assessment. Specifically, we instructed the geriatricians to discuss and address patients' physical and psychological symptom concerns, comorbid conditions and polypharmacy, cognitive issues, availability of social supports, functional impairments, use of coping strategies, and illness understanding. After each visit, the geriatricians communicated (either in person or via phone/email) their findings with the patient's oncology team.

\section{Usual Care}

Participants receiving usual care could meet with a geriatrician upon request by the oncologist, patient, and/or family. However, no patients receiving usual care ultimately met with a geriatrician. All patients, regardless of group assignment, continued to receive routine oncology care throughout the study period.

\section{Study Measures}

\section{Sociodemographic and Clinical Characteristics}

Participants completed baseline study measures before randomization, and were asked to self-report their sex, race, relationship status, employment status, education level, annual income, and comorbid conditions. Information on age and cancer type was obtained from the EHR.

\section{Patient-Reported Outcomes}

At baseline and week 12, participants self-reported their QoL, symptoms, functional outcomes, communication confidence, and illness perceptions. To assess QoL, we used the Functional Assessment of Cancer TherapyGeneral (FACT-G), which has been validated for use in older adults with cancer. ${ }^{27}$ FACT-G consists of subscales assessing well-being across 4 domains (physical, functional, emotional, and social) during the preceding 7 days. Scores range from 0 to 108, with higher scores indicating better QoL.

To assess symptoms, we used the self-administered Edmonton Symptom Assessment System - Revised (ESAS-r), a validated tool for measuring symptoms. ${ }^{23}$ Each specific symptom is scored from 0 to $10(0=a b-$ sence of the symptom; $10=$ worst possible severity). Consistent with earlier research, we categorized the severity of ESAS scores as 0 (none), 1 to 3 (mild), 4 to 6 (moderate), and 7 to 10 (severe), and computed composite ESAS-physical and ESAS-total symptom scores. ${ }^{28,29}$ To measure depression symptoms, we used the 15-item Geriatric Depression Scale (GDS), with higher scores reflecting worse depression symptoms (range, 0-15, with scores $>4$ indicating the presence of clinically significant depression symptoms).

To assess functional outcomes, we asked patients about their activities of daily living (ADLs) and instrumental activities of daily living (IADLs). For ADLs, we used a subscale from the Medical Outcomes Study to determine the number of independent ADLs (range, $0-10) .{ }^{31}$ For IADLs, we used a subscale from the Multidimensional Functional Assessment Questionnaire from the Older Americans Resources and Services Program to determine the number of independent IADLs (range, $0-7){ }^{32}$

To assess communication confidence, we used the 10-item Perceived Efficacy in Patient-Physician Interactions Questionnaire, a tool validated for use in older patients. Scores range from 10 to 50, with higher scores reflecting patients' greater confidence in the ability to communicate and seek help from providers. ${ }^{33}$

To assess illness perceptions, we used the Brief Illness Perception Questionnaire, a validated instrument. Scores range from 0 to 80 , with higher scores indicating a more threatening perception of the illness. $^{34}$

\section{Acceptability of the Intervention}

As part of the week 12 assessment, we asked patients assigned to the intervention to complete a written survey inquiring about the timing and utility of the intervention. Specifically, we asked patients about their perceptions of the visit number and length and whether they considered the visit with the geriatrician helpful.

\section{Statistical Analysis}

The primary endpoint was feasibility. We predefined the intervention as feasible if at least $70 \%$ of patients enrolled in the study and at least $75 \%$ of living patients completed study visits and surveys. To evaluate the acceptability of the intervention, we tabulated intervention participants' responses to the acceptability survey.

Secondary endpoints included evaluation of the effect size of the transdisciplinary intervention for improving QoL, symptom burden, functional outcomes, communication confidence, and illness perceptions. For each of these outcomes, we calculated effect sizes (ES) (Cohen's $d$ ) for changes from baseline to week 12 ([mean change score (intervention arm) - mean change score (usual care arm)] $/ \mathrm{SD}_{\text {pooled }}$ ), where 0.2 indicated a 
small effect, 0.5 a medium effect, and 0.8 a large effect. ${ }^{35}$ Because of the pilot nature of this study, we used available case analysis.

\section{Results}

\section{Participant Characteristics}

Table 1 displays baseline characteristics for each of the study arms. Patients had a median age of 72.26 years (range, ages 65.22-91.84 years), and most were white (98.4\%), male (54.8\%), married $(66.1 \%)$, and retired (79.0\%). The most common cancer types were non-small cell lung cancer (38.7\%), pancreatic cancer $(22.6 \%)$, and hepatobiliary cancer (16.1\%). Patients were an average of 4.40 weeks (SD, 2.06) from their diagnosis with advanced cancer, and $74.2 \%$ reported at least 1 comorbid condition.

\section{Baseline Patient-Reported Outcomes}

Baseline patient-reported outcomes were balanced between randomization arms (Table 2). Patients reported an average of 3.28 (SD, 3.15) moderate-severe symptoms at baseline, with $73.3 \%$ reporting at least 1 moderatesevere symptom and $24.2 \%$ having clinically significant depression symptoms. At baseline, patients reported an average of 4.26 (SD, 3.07) independent ADLs and 5.52 (SD, 1.80) independent IADLs, with only 1 patient reporting independence on all ADLs/IADLs.

Feasibility and Acceptability of the Intervention We enrolled $55.9 \%(62 / 111)$ of patients approached (Figure 2). The most common reasons for refusing study participation were feeling too ill $(20.7 \%)$ and not being interested in the research (18.9\%). Among patients assigned to the intervention, $82.1 \%(23 / 28)$ attended the first geriatric visit and $79.6 \%$ (39/49) attended both visits. Overall, $89.7 \%$ completed the baseline and week 12 study surveys.

Participants found the intervention acceptable, with $73.3 \%(11 / 15)$ reporting that the number of visits with the geriatrician was the right amount, 68.8\% (11/16) reporting that the length of the visit was the right amount, and $62.5 \%(10 / 16)$ reporting that the visit was helpful (Figure 3).

\section{Intervention Effect Sizes for Improving \\ Patient-Reported Outcomes}

We found that the intervention had small to medium effect sizes (ES) for improving many patient-reported outcomes (Table 3). From baseline to week 12, patients assigned to the intervention had less decrement in their QoL than those assigned to usual care (mean change -0.77 vs -3.84 ; $E S=0.21$ ). In addition, those assigned to intervention experienced decreased severity of their

\section{Table 1. Baseline Participant Characteristics}

\begin{tabular}{|c|c|c|}
\hline Characteristic & $\begin{array}{l}\text { Usual Care } \\
\text { n (\%) }\end{array}$ & $\begin{array}{c}\text { Geriatric } \\
\text { Intervention } \\
\mathbf{n}(\%)\end{array}$ \\
\hline Total, $\mathrm{n}$ & 32 & 30 \\
\hline Age, mean (SD), y & $73.68(6.22)$ & $73.94(6.51)$ \\
\hline \multicolumn{3}{|l|}{ Sex } \\
\hline Male & $18(56.2)$ & $16(53.3)$ \\
\hline Female & $14(43.8)$ & $14(46.7)$ \\
\hline \multicolumn{3}{|l|}{ Race } \\
\hline White & $32(100.0)$ & $29(96.7)$ \\
\hline Black & $0(0.0)$ & $1(3.3)$ \\
\hline \multicolumn{3}{|l|}{ Relationship status } \\
\hline Married & $20(62.5)$ & $21(70.0)$ \\
\hline Widowed & $3(9.4)$ & $6(20.0)$ \\
\hline Divorced & $4(12.5)$ & $2(6.7)$ \\
\hline Single & $5(15.6)$ & $1(3.3)$ \\
\hline \multicolumn{3}{|l|}{ Employment status } \\
\hline Full-time & $5(15.6)$ & $6(20.0)$ \\
\hline Part-time & $1(3.1)$ & $1(3.3)$ \\
\hline Retired & $26(81.2)$ & $23(76.7)$ \\
\hline \multicolumn{3}{|l|}{ Education level } \\
\hline Less than college graduate & $16(50.0)$ & $19(63.3)$ \\
\hline College graduate and above & $15(46.9)$ & $10(33.3)$ \\
\hline \multicolumn{3}{|l|}{ Annual income, \$USD } \\
\hline$<\$ 60,000$ & $12(42.9)$ & $8(32.0)$ \\
\hline$\geq \$ 60,000$ & $16(57.1)$ & $17(68.0)$ \\
\hline \multicolumn{3}{|l|}{ Cancer type } \\
\hline NSCLC & $12(37.5)$ & $12(40.0)$ \\
\hline Pancreatic & $4(12.5)$ & $10(33.3)$ \\
\hline Hepatobiliary & $6(18.8)$ & $4(13.3)$ \\
\hline Gastroesophageal & $4(12.5)$ & $2(6.7)$ \\
\hline Colorectal & $4(12.5)$ & $1(3.3)$ \\
\hline SCLC & $2(6.2)$ & $1(3.3)$ \\
\hline $\begin{array}{l}\text { Weeks since advanced cancer } \\
\text { diagnosis, mean (SD) }\end{array}$ & $4.64(2.02)$ & $4.15(2.10)$ \\
\hline \multicolumn{3}{|l|}{ Comorbid conditions } \\
\hline Chronic lung disease & $8(25.0)$ & $8(26.7)$ \\
\hline Diabetes & $8(25.0)$ & $7(23.3)$ \\
\hline Liver problem & $10(31.2)$ & $3(10.0)$ \\
\hline Kidney problem & $4(12.5)$ & $6(20.0)$ \\
\hline Stroke & $2(6.2)$ & $7(23.3)$ \\
\hline Circulation trouble & $1(3.1)$ & $5(16.7)$ \\
\hline Rheumatologic condition & $2(6.2)$ & $3(10.0)$ \\
\hline Heart attack & $3(9.4)$ & $0(0.0)$ \\
\hline Heart failure & $0(0.0)$ & $0(0.0)$ \\
\hline
\end{tabular}

Abbreviations: NSCLC, non-small cell lung cancer; SCLC, small cell lung cancer. 


\begin{tabular}{|c|c|c|}
\hline Patient-Reported Outcome & $\begin{array}{l}\text { Usual Care } \\
\text { Mean (SD) }\end{array}$ & $\begin{array}{l}\text { Intervention } \\
\text { Mean (SD) }\end{array}$ \\
\hline Total, n & 32 & 30 \\
\hline \multicolumn{3}{|l|}{ Quality of life } \\
\hline FACT-G total & $78.94(12.65)$ & $76.50(16.78)$ \\
\hline \multicolumn{3}{|l|}{ Symptoms } \\
\hline ESAS physical score & 17.75 (13.39) & $17.70(13.65)$ \\
\hline ESAS total score & $24.78(18.23)$ & $27.63(19.88)$ \\
\hline $\begin{array}{l}\text { Number of moderate-severe } \\
\text { ESAS symptoms }\end{array}$ & $2.91(2.87)$ & $3.71(3.44)$ \\
\hline GDS score & $3.13(2.69)$ & $3.73(3.48)$ \\
\hline \multicolumn{3}{|l|}{ Functional outcomes } \\
\hline Number of independent ADLs & $4.06(2.81)$ & $4.47(3.36)$ \\
\hline Number of independent IADLs & $5.75(1.44)$ & $5.27(2.12)$ \\
\hline \multicolumn{3}{|c|}{ Communication confidence and illness perceptions } \\
\hline $\begin{array}{l}\text { Perceived efficacy in } \\
\text { patient-physician interactions }\end{array}$ & $47.00(5.81)$ & $45.87(5.17)$ \\
\hline $\begin{array}{l}\text { Brief illness perception } \\
\text { questionnaire }\end{array}$ & $36.44(11.30)$ & $41.97(12.07)$ \\
\hline
\end{tabular}

Abbreviations: ADLs, activities of daily living; ESAS, Edmonton Symptom Assessment System; FACT-G, Functional Assessment of Cancer TherapyGeneral; GDS, Geriatric Depression Scale; IADLs, instrumental activities of daily living.

ESAS total scores (mean change, -0.47 vs +5.72 ; ES $=$ 0.35 ), reduction in the number of moderate-severe ESAS symptoms (mean change, -0.69 vs +1.04 ; $\mathrm{ES}=0.58$ ), and lower GDS scores (mean change, -0.47 vs +0.58 ; $\mathrm{ES}=0.36)$ compared with patients assigned to usual care. Patients assigned to intervention also reported improvements in their communication confidence (mean change, +1.06 vs -0.80 ; $\mathrm{ES}=0.38$ ).

\section{Discussion}

In this pilot RCT, we investigated the feasibility and acceptability of a transdisciplinary intervention targeting patients' geriatric and palliative care needs, and we estimated the effect size of this intervention for improving patient-reported outcomes in older adults with cancer. We enrolled more than half of the patients that were approached, and more than three-fourths of patients assigned to the intervention attended the geriatrician visits. In addition, most patients found the intervention acceptable and helpful. Notably, the intervention demonstrated encouraging effect size estimates for enhancing patients' QoL, physical and psychological symptoms, and communication confidence.

Importantly, our work highlights the need for interventions to target the palliative care and geriatricspecific issues unique to older adults with cancer. Consistent with earlier work, at baseline, patients in our sample averaged $>3$ moderate-severe symptoms, with

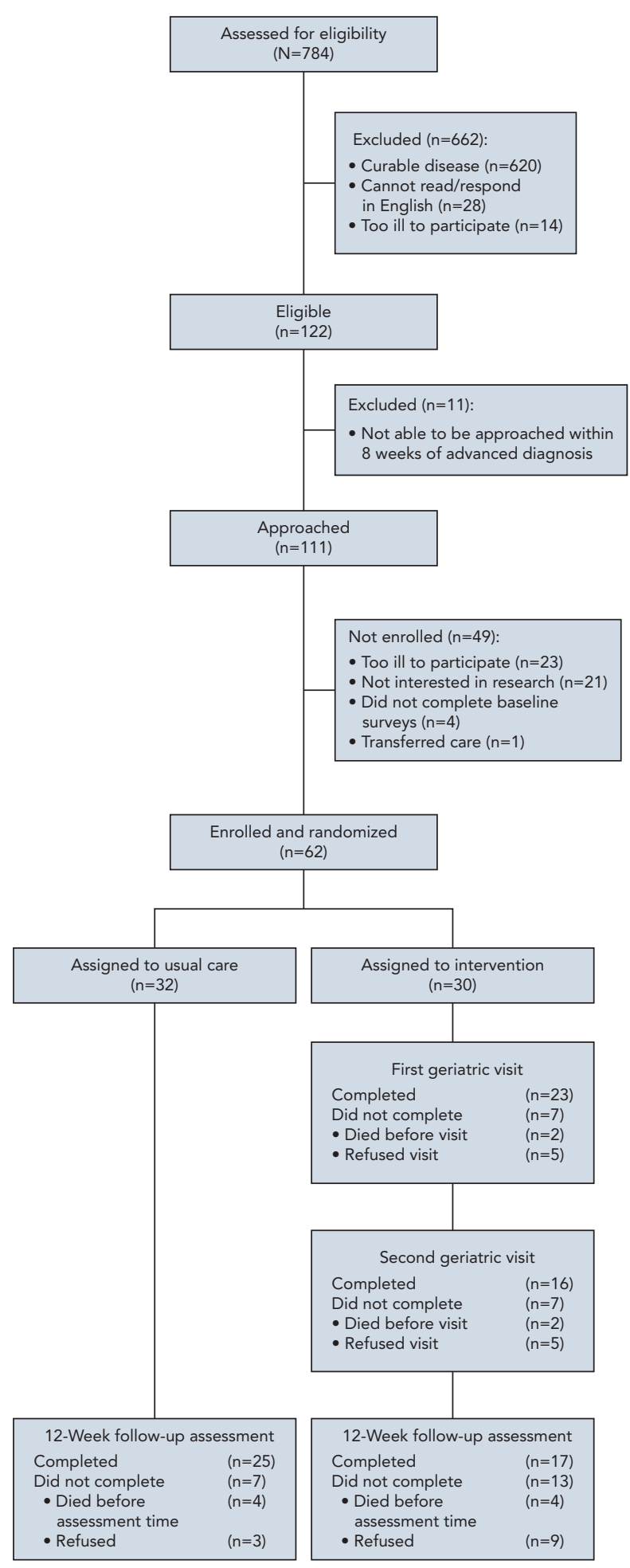

Figure 2. CONSORT diagram.

nearly three-fourths of patients reporting at least 1 moderate-severe symptom and one-fourth reporting depression symptoms. ${ }^{7,8,10,11,36}$ In addition, three-fourths of patients had at least 1 comorbid condition, and only 


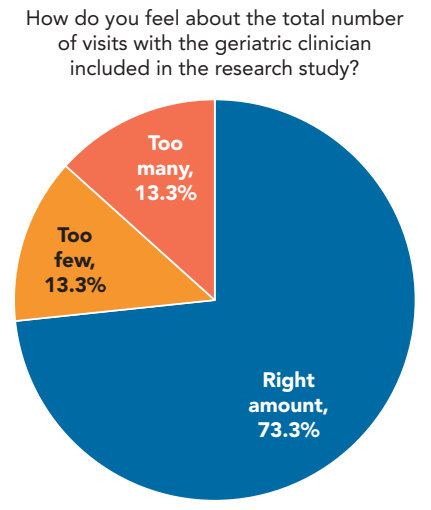

How do you feel about the amount of time each geriatric clinician visit lasted?

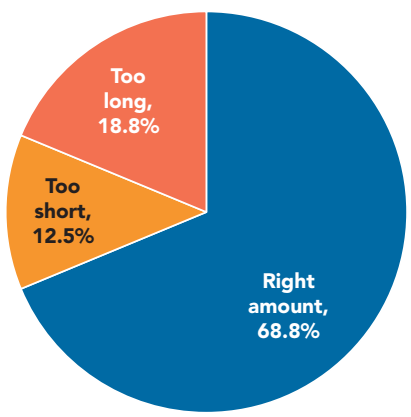

How helpful were geriatric clinician visits?

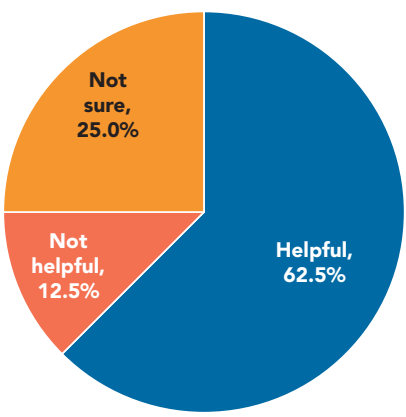

Figure 3. Intervention acceptability ratings.

1 patient in our cohort reported being independent with all ADLs/IADLs, which is also consistent with previous research. ${ }^{9,36}$ With such high baseline symptom burden, pervasive comorbid conditions, and impaired functional status, our findings underscore the tremendous potential for efforts such as this transdisciplinary intervention to enhance care outcomes for the geriatric oncology population.

This study represents our first attempt to integrate a transdisciplinary intervention addressing patients' geriatric-specific and palliative care needs into the care of older adults with cancer. This intervention ensures that older adults with cancer receive focused attention to their complex combination of medical and psychosocial issues by incorporating geriatricians into their outpatient oncologic care. Although we enrolled more than half of patients approached, we did not meet our goal of at least $70 \%$. This likely resulted from the profound illness severity of the study population, given that nearly half who declined reported feeling too ill to participate. In future iterations of this work, we will adapt our study procedures to further remove barriers and ensure that patients can participate; this cohort represents a high-risk group who may particularly benefit from interventions such as this. ${ }^{18,19}$ Our geriatricians care for patients at a clinic that is not integrated within the cancer center, and moving forward we will work to co-locate our geriatric interventions within the

\section{Table 3. ES Estimates of the Intervention for Improving Patient-Reported Outcomes}

\begin{tabular}{|c|c|c|c|}
\hline Patient-Reported Outcome & $\begin{array}{l}\text { Usual Care } \\
\text { Mean } \Delta \text { (SD) }\end{array}$ & $\begin{array}{l}\text { Intervention } \\
\text { Mean } \Delta \text { (SD) }\end{array}$ & Cohen's d ES \\
\hline \multicolumn{4}{|l|}{ Quality of life } \\
\hline FACT-G total & $-3.84(15.75)$ & $-0.77(14.10)$ & 0.21 \\
\hline \multicolumn{4}{|l|}{ Symptoms } \\
\hline ESAS physical score & $3.96(10.69)$ & $0.06(15.72)$ & 0.29 \\
\hline ESAS total score & $5.72(13.94)$ & $-0.47(20.61)$ & 0.35 \\
\hline Number of moderate-severe ESAS symptoms & $1.04(2.82)$ & $-0.69(3.18)$ & 0.58 \\
\hline GDS score & $0.58(2.90)$ & $-0.47(2.98)$ & 0.36 \\
\hline \multicolumn{4}{|l|}{ Functional outcomes } \\
\hline Number of independent ADLs & $-0.68(1.93)$ & $-0.88(3.67)$ & 0.07 \\
\hline Number of independent IADLs & $-0.24(1.48)$ & $-0.19(1.22)$ & 0.04 \\
\hline \multicolumn{4}{|l|}{ Communication confidence and illness perceptions } \\
\hline $\begin{array}{l}\text { Perceived efficacy in patient-physician } \\
\text { interactions }\end{array}$ & $-0.80(6.49)$ & $1.06(2.54)$ & 0.38 \\
\hline Brief illness perception questionnaire & $0.96(9.95)$ & $0.88(12.77)$ & 0.01 \\
\hline
\end{tabular}

Abbreviations: ADLs, activities of daily living; ES, effect size; ESAS, Edmonton Symptom Assessment System; FACT-G, Functional Assessment of Cancer TherapyGeneral; GDS, Geriatric Depression Scale; IADLs, instrumental activities of daily living; mean $\Delta$, mean change.

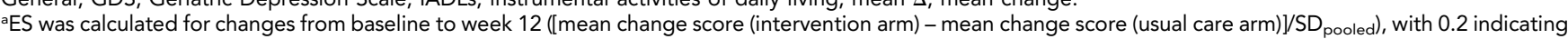
a small effect, 0.5 a medium effect, and 0.8 a large effect. 
cancer center to minimize the burden of additional travel and extra visits. We also chose an ambitious enrollment goal, which several studies of geriatricspecific interventions have similarly not achieved. ${ }^{37-39}$ Despite not meeting our prespecified enrollment rate, most participants completed the study visits and surveys, and most found the intervention acceptable. Therefore, our findings highlight the feasibility of delivering a transdisciplinary intervention focused on the geriatric-specific issues and palliative care needs of older patients with cancer, yet we also learned important lessons to inform future iterations of this work to enhance care quality and outcomes for the geriatric oncology population, a rapidly growing group of patients needing interventions tailored to their distinct needs.

We found that the transdisciplinary intervention resulted in favorable effect size estimates for improving several important patient-reported outcomes. During the study period, QoL scores deteriorated over time in both study arms, as expected from a population with advanced cancer, ${ }^{15-17}$ but we observed better preservation of QoL in the intervention arm. Notably, those in the intervention arm reported improvements in their symptom burden and communication confidence during the study period, but those assigned to usual care experienced worsening of these outcomes. Patients' symptoms and QoL represented direct targets of the transdisciplinary intervention, which could explain the favorable results for these outcomes. However, the findings for enhancing communication confidence are hypothesis-generating, potentially related to the additional attention patients received from geriatricians trained to comprehensively address their unique needs while empowering them to openly discuss their concerns. Importantly, patients' QoL, symptom burden, and communication confidence represent patient-centered outcomes critical to consider when designing interventions for older adults with cancer, and our results underscore the potential for targeted supportive care interventions to address these outcomes. ${ }^{12,40}$

Several limitations warrant discussion. First, this trial was conducted at an academic institution with limited sociodemographic diversity, reducing the generalizability of our findings. Second, data were not available regarding certain factors that could influence the impact of the intervention, including patients' social supports, cognition, and receipt of other services, such as psychiatry or physical and occupational therapy. Future work should investigate whether these and other important factors, such as cancer type and use of certain coping strategies, influence the effects of this intervention on patient outcomes. Notably, our study cohort included a heterogeneous group of patients with incurable gastrointestinal or lung cancer, who likely received different treatment regimens, and future research will need to investigate how the disease and treatment influence the impact of this intervention. In addition, we did not audiorecord study visits, and therefore we do not have specific information regarding factors discussed at each study visit or amount of variation in the content of each visit. We also lack information about clinicians' perceptions regarding the utility of the transdisciplinary intervention, and future efforts to fully integrate geriatricians into the cancer care team should consider these important perspectives.

\section{Conclusions}

In this study, we sought to determine the feasibility and acceptability of a transdisciplinary intervention designed to address the geriatric-specific and palliative care needs of older adults with advanced cancer. Although we did not meet our predetermined feasibility goal of enrolling $>70 \%$ of patients approached, we found that more than half of patients we asked to participate enrolled, and most completed all study visits and surveys, with the majority finding the intervention acceptable and helpful. Notably, we found encouraging effect size estimates for the transdisciplinary intervention to improve patients' QoL, physical and psychological symptoms, and communication confidence. In addition, our data highlight older patients' substantially high symptom burden, comorbidity, and functional impairments, thus underscoring the critical importance of efforts to address the geriatric and palliative care needs of older adults with cancer. Collectively, these results support the need for a larger RCT to show the efficacy of this care model to enhance care outcomes for the geriatric oncology population.

Submitted August 24, 2019; accepted for publication December 4, 2019

Previous presentation: Presented in abstract form at the 2019 ASCO Annual Meeting; May 31-June 4, 2019; Chicago, Illinois (J Clin Oncol 2019;37 [Suppl]:Abstract 11549).

Author contributions: All authors made substantial contributions to conception and design, acquisition of data, or analysis and interpretation of data. All were involved in drafting the article or revising it critically for important intellectual content. All provided final approval of the manuscript and agree to be accountable for all aspects of the work.

Disclosures: Dr. Greer has disclosed that he recieves grant/research support from and is a consultant for Gaido Health/BCG Digital Ventures. The remaining authors have disclosed that they have not received any financial consideration from any person or organization to support the preparation, analysis, results, or discussion of this article.

Funding: Dr. Temel has disclosed funding from NCl (K24 CA181253). Dr. Nipp has disclosed funding from NIH/National Institute on Aging (R03AG053314)

Correspondence: Ryan D. Nipp, MD, MPH, Department of Medicine, Division of Hematology and Oncology, Massachusetts General Hospital Cancer Center, 55 Fruit Street, Yawkey 7B, Boston, MA 02114 Email: rnipp@mgh.harvard.edu 


\section{References}

1. Yancik R. Population aging and cancer: a cross-national concern. Cancer J 2005;11:437-441.

2. Patel SS, Nelson R, Sanchez J, et al. Elderly patients with colon cancer have unique tumor characteristics and poor survival. Cancer 2013;119: 739-747.

3. Neugut Al, Matasar M, Wang X, et al. Duration of adjuvant chemotherapy for colon cancer and survival among the elderly. J Clin Oncol 2006;24: 2368-2375.

4. Sargent DJ, Goldberg RM, Jacobson SD, et al. A pooled analysis of adjuvant chemotherapy for resected colon cancer in elderly patients. N Engl J Med 2001;345:1091-1097.

5. Mor V, Allen S, Malin M. The psychosocial impact of cancer on older versus younger patients and their families. Cancer 1994;74(7 Suppl):2118-2127.

6. Nipp RD, Greer JA, El-Jawahri A, et al. Coping and prognostic awareness in patients with advanced cancer. J Clin Oncol 2017;35:2551-2557.

7. Cheung WY, Le LW, Gagliese L, et al. Age and gender differences in symptom intensity and symptom clusters among patients with metastatic cancer. Support Care Cancer 2011;19:417-423.

8. Linden W, Vodermaier A, Mackenzie R, et al. Anxiety and depression after cancer diagnosis: prevalence rates by cancer type, gender, and age. J Affect Disord 2012;141:343-351.

9. Derks MG, de Glas NA, Bastiaannet $E$, et al. Physical functioning in older patients with breast cancer: a prospective cohort study in the TEAM trial. Oncologist 2016;21:946-953.

10. Brighi N, Balducci L, Biasco G. Cancer in the elderly: is it time for palliative care in geriatric oncology? J Geriatr Oncol 2014;5:197-203.

11. Blank TO, Bellizzi KM. A gerontologic perspective on cancer and aging Cancer 2008;112(11 Suppl):2569-2576.

12. Mohile SG, Velarde C, Hurria A, et al. Geriatric assessment-guided care processes for older adults: a Delphi consensus of geriatric oncology experts. J Natl Compr Canc Netw 2015;13:1120-1130.

13. Dotan E, Walter LC, Baumgartner J, et al. NCCN Clinical Practice Guidelines in Oncology: Older Adult Oncology. Version 1.2019. Accessed August 24, 2019. To view the most recent version, visit NCCN.org.

14. Mohile SG, Dale W, Somerfield MR, et al. Practical assessment and management of vulnerabilities in older patients receiving chemotherapy: ASCO guideline for geriatric oncology. J Clin Oncol 2018;36:2326-2347.

15. Temel JS, Greer JA, Muzikansky A, et al. Early palliative care for patients with metastatic non-small-cell lung cancer. N Engl J Med 2010;363: 733-742.

16. Zimmermann C, Swami N, Krzyzanowska M, et al. Early palliative care for patients with advanced cancer: a cluster-randomised controlled trial. Lancet 2014;383:1721-1730.

17. Temel JS, Greer JA, El-Jawahri A, et al. Effects of early integrated palliative care in patients with lung and GI cancer: a randomized clinical trial. $\mathrm{J}$ Clin Oncol 2017;35:834-841.

18. Nipp RD, El-Jawahri A, Traeger $L$, et al. Differential effects of early palliative care based on the age and sex of patients with advanced cancer from a randomized controlled trial. Palliat Med 2018;32:757-766.

19. Nipp RD, Greer JA, El-Jawahri A, et al. Age and gender moderate the impact of early palliative care in metastatic non-small cell lung cancer. Oncologist 2016;21:119-126.

20. Stuck AE, Siu AL, Wieland GD, et al. Comprehensive geriatric assessment: a meta-analysis of controlled trials. Lancet 1993;342:1032-1036.

21. Mohile S, Dale W, Magnuson A, et al. Research priorities in geriatric oncology for 2013 and beyond. Cancer Forum 2013;37:216-221.
22. Dankwa-Mullan I, Rhee KB, Stoff DM, et al. Moving toward paradigmshifting research in health disparities through translational, transformational, and transdisciplinary approaches. Am J Public Health 2010; 100(Suppl 1):S19-24.

23. Bruera E, Kuehn N, Miller MJ, et al. The Edmonton Symptom Assessment System (ESAS): a simple method for the assessment of palliative care patients. J Palliat Care 1991;7:6-9.

24. Kroenke K, Spitzer RL, Williams JB, et al. An ultra-brief screening scale for anxiety and depression: the PHQ-4. Psychosomatics 2009;50: 613-621.

25. Ferguson M, Capra S, Bauer J, et al. Development of a valid and reliable malnutrition screening tool for adult acute hospital patients. Nutrition 1999;15:458-464.

26. Saliba D, Elliott M, Rubenstein LZ, et al. The Vulnerable Elders Survey: a tool for identifying vulnerable older people in the community. J Am Geriatr Soc 2001:49:1691-1699.

27. Overcash J, Extermann M, Parr J, et al. Validity and reliability of the FACT-G scale for use in the older person with cancer. Am J Clin Oncol 2001;24:591-596.

28. Nipp RD, El-Jawahri A, Moran SM, et al. The relationship between physical and psychological symptoms and health care utilization in hospitalized patients with advanced cancer. Cancer 2017;123:4720-4727.

29. Nipp RD, El-Jawahri A, D'Arpino SM, et al. Symptoms of posttraumatic stress disorder among hospitalized patients with cancer. Cancer 2018; 124:3445-3453.

30. D'Ath P, Katona P, Mullan E, et al. Screening, detection and management of depression in elderly primary care attenders. I: the acceptability and performance of the 15 item Geriatric Depression Scale (GDS15) and the development of short versions. Fam Pract 1994;11:260-266.

31. Stewart $A L$, Kamberg $C$. Physical functioning measures. In: Stewart $A L$, Ware JE Jr, eds. Measuring Functioning and Well-Being: The Medical Outcomes Study Approach. Durham, NC:Duke University Press; 1992: 86-101.

32. Fillenbaum GG, Smyer MA. The development, validity, and reliability of the OARS Multidimensional Functional Assessment Questionnaire. J Gerontol 1981;36:428-434.

33. Maly RC, Frank JC, Marshall GN, et al. Perceived efficacy in patientphysician interactions (PEPPI): validation of an instrument in older persons. J Am Geriatr Soc 1998;46:889-894.

34. Broadbent E, Petrie KJ, Main J, et al. The brief illness perception questionnaire. J Psychosom Res 2006;60:631-637.

35. Cohen J. A power primer. Psychol Bull 1992;112:155-159.

36. Pandya C, Magnuson A, Flannery M, et al. Association between symptom burden and physical function in older patients with cancer. J Am Geriat Soc 2019;67:998-1004.

37. Demark-Wahnefried W, Clipp EC, Morey MC, et al. Lifestyle intervention development study to improve physical function in older adults with cancer: outcomes from Project LEAD. J Clin Oncol 2006;24:3465-3473.

38. Oldervoll LM, Loge JH, Lydersen S, et al. Physical exercise for cancer patients with advanced disease: a randomized controlled trial. Oncologist 2011;16:1649-1657.

39. Horgan AM, Leighl NB, Coate L, et al. Impact and feasibility of a comprehensive geriatric assessment in the oncology setting: a pilot study. Am $\mathrm{J}$ Clin Oncol 2012;35:322-328.

40. Nipp RD, Yao N, Lowenstein LM, et al. Pragmatic study designs for older adults with cancer: report from the U13 conference. J Geriatr Oncol 2016 $7: 234-241$. 